\title{
Channel Analysis Based on Received Signal Strength in Wireless Body Sensor Network Systems
}

\author{
Jina Hong, Woosik Lee and Namgi Kim* \\ Department of Computer Science, Kyonggi University \\ \{jin-a,wslee,ngkim\}@kgu.ac.kr
}

\begin{abstract}
In wireless body sensor network (WBSN) environments, diverse factors can affect channel environments. For example, the condition of the channel can be changed due to sensor mobility resulting from movements of the experimenter. In addition, the placement of the sensors can change the channel. In this paper, we analyzed the impact of different sensor placements and body movements on the channel environment by measuring the received signal strength indication (RSSI) and the packet delivery rate (PDR). Diverse experiments were conducted with real sensor devices in an office environment. The sensors were placed on the subject's stomach, back, and ankle, and data were obtained while the subject stood, walked, and ran. The experimental results showed that the RSSI decreased in accordance with an increase in the activity of the experimenter. They also revealed that the RSSI deviation varied depending on the placement of the sensors.
\end{abstract}

Keywords: wireless body sensor network, received signal strength indication, transmission power level

\section{Introduction}

As wireless sensor network (WSN) technology has developed, devices have become smaller, and more demands for WBSN are created. In response to such demands, wireless body sensor network (WBSN) technology is constantly being updated. WBSN technology is used in various areas of health care to improve people's quality of life. WBSN sensors have been inserted in clothes and even transplanted under the skin. However, there are many limitations in applying existing WSNs to WBSN technology [1,2]. As the network is formed inside the human body, smaller and fewer nodes have to be used, and the nodes have to perform many more tasks. From a network topology point of view, WSNs are fixed. In contrast, WBSNs can have a variety of topologies due to the movement of the body. Moreover, energy consumption is greatly influenced by activity. From a power demand point of view, WBSNs experience more difficulties in energy supply compared to WSNs, and they have to be operated at low power. Importantly, WBSNs differ from WSNs in data rates, propagation, biocompatibility, and the impact of data loss. Therefore, it is necessary to consider factors that dynamically alter channel environments in WBSNs.

In a WBSN, the received signal strength indication (RSSI) values can differ because of the previously mentioned channel conditions. The transmission power level (TPL) and the RSSI values change according to the type of radio chips used. This study varied the placement of sensors, human movements, and the TPL and performed a WBSN experiment to analyze the

${ }^{*}$ Corresponding author: Namgi Kim. 
RSSI values, RSSI deviations, and the package data rate (PDR) under different channel environments.

\section{Related work}

The WBSN environment can be dynamic according to the placement of the sensors, human activity, the surrounding environment, and the location [3-4]. Among these factors, this study focuses on sensor placements and human activity. As the sensor nodes are placed on the human body, they can move periodically with the movement of the body, and their locations can change. Therefore, this study focused on analyzing a dynamic WBSN environment and channel links through experiments.

Many studies have analyzed dynamic WBSN environments [5-8]. Qi [5] analyzed he accuracy, latency, and battery life in a WBSN. This research considered a wide range of activities, including not only standing, walking, and running but also lying and riding. However, the sensor placements were limited to the subject's wrist and ankle. Therefore, it is necessary to research difference of deviation by varying the placement of the sensors

Lee [6] conducted various experiments with 10 sensors placed on the subject's stomach, back, left and right ankles, thighs, wrists, and forearms to analyze the channel links. However, the experimenter was fixed in a standing position. For WBSNs in dynamic environments, we need to consider not only a standing position but also other positions and movements. The experiments conducted by Qi and Lee used a CC2420 radio chip. However, the frequency, transmission power control level, output power range, and supply voltage [7] of the CC2420 chip differs from those of the CC1000 module. Therefore, it is necessary to analyze dynamic WBSN channel links using a CC1000 module.

Finally, Natarajan [8] placed sensors on various locations similar to the study by Lee. The study involved 4 experimenters, and the authors observed their natural movements in an experimental environment. In contrast to earlier research, this study classified movements into standing, walking, and running and examined the impact of each movement on the RSSI and PDR. Natarajan used both CC1000 and CC2420 [9] radio chips. However, a limitation of the study was that the authors set the TPL to default according to the experimental locations. In contrast, the current study also analyzed the effect of the TPL.

\section{Analysis of sensor placements}

This section analyzes the effect of the sensor node placements and body movements on the WBSN. Table 1 provides information on the placement of the sensors and the experimental environment. In the current study, the body was divided into static and dynamic areas based on the placement of the sensors. The static areas were the subject's stomach and back, both of which show less movement than other locations. The dynamic areas included sensor nodes the subject's arms and legs where the locations of the sensor nodes change according to the movement of the body. The sensor nodes placed on dynamic areas are more influenced by body movements than those placed on static areas because of obstacles between the sink nodes and the sensor nodes. In addition, the distance between the nodes changes according to the movement of the body. 
Table 1. Placement of the sensors and the experimental environment

\begin{tabular}{|c|c|c|c|}
\hline \multirow{2}{*}{ Sensor Placements } & \multicolumn{3}{|c|}{ Factors } \\
\cline { 2 - 4 } & Area & Distance & Obstacle \\
\hline Stomach & Static & Short & No \\
\hline Back & Static & Middle & Yes \\
\hline Forearm & Dynamic & Middle & Yes \\
\hline Wrist & Dynamic & Long & Yes \\
\hline Thigh & Dynamic & Long & Yes \\
\hline Ankle & Dynamic & Long & Yes \\
\hline
\end{tabular}

\section{Experimental environment}

To create a body sensor system channel, this study conducted experiments using an actual sensor mote: Cricket Mote produced by Crossbow Technology [10]. The radio chip of Cricket Mote uses a CC1000 module [11], with a frequency range of $868 \mathrm{MHz}$. The TPL range is 22, with levels from 1 to 22 .

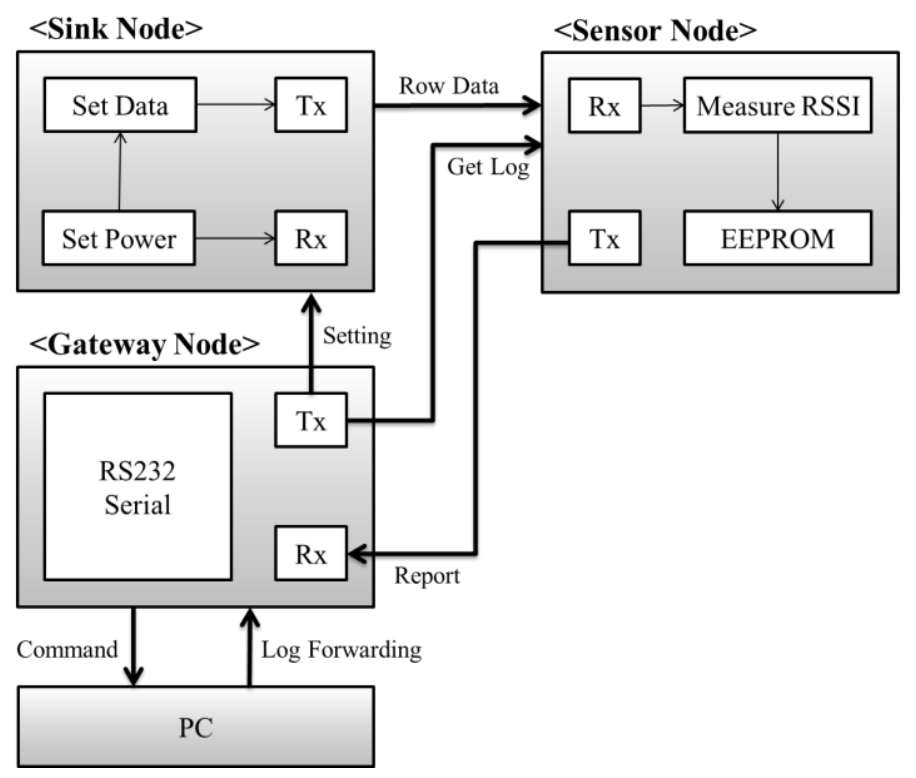

Figure 4. System structure

Figure 4 shows the system structure, which consists of a PC, a gateway node, a sink node, and a sensor node. The system is operated as follows using the already mentioned mode: the user commands are transmitted from the PC to the gateway node through an RS232 serial port. The gateway node sends the command to a sink node. Based on the command received, the sink node sets the current power level, timer, and log data. The sink node transmits the data to the sensor node according to a set timer, and the sensor node measures the RSSI value of the received data and saves the $\log$ on EEPROM. Later, when the gateway node asks for a $\log$ record from the sensor node through the Get Log command, the sensor node transmits the measured value saved in EEPROM to the gateway node using Report Message. The gateway node then forwards the log. 


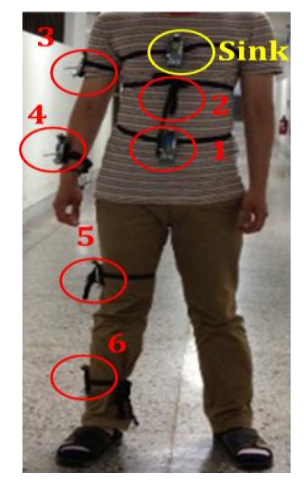

\section{Figure 5. Locations of sink node and sensor nodes on the body}

To consider various channel environments, this study decided specific locations for sensor node placements and specific movements for the experimenter. Figure 5 shows the placement of the sensors. The sensor nodes were placed on six locations: stomach, back, forearm, wrist, thigh, and ankle. The sink node was placed on the subject's chest.

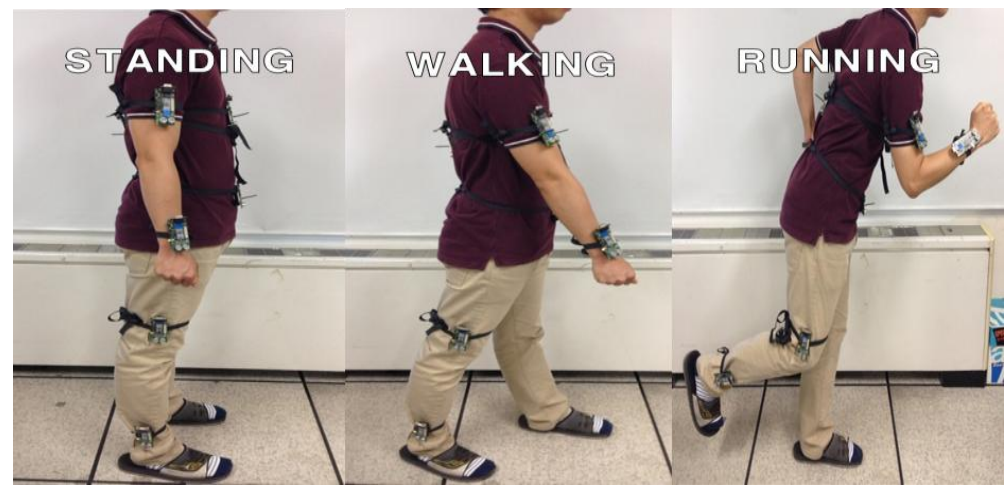

Figure 6. Movements: Standing, walking, running

Figure 6 shows the human movements studied in the experiment. The experimenter moved with six sensor nodes and one sink node on his body. Three movements were considered: standing, walking, and running.

A room was selected as the experimental environment in the present study because it is appropriate for analyzing the characteristics of body movements and the effects of the locations of the sensors. Unlike a large playground environment, the small size of the room means that multipath fading exerts a greater effect, thereby giving higher PDR and RSSI values and providing better experiment results.

\section{Experimental Result}

This study analyzed the experimental results according to the experimenter's movements and the placement of the sensor nodes. The analysis considered RSSI, TRL, and PDR. 


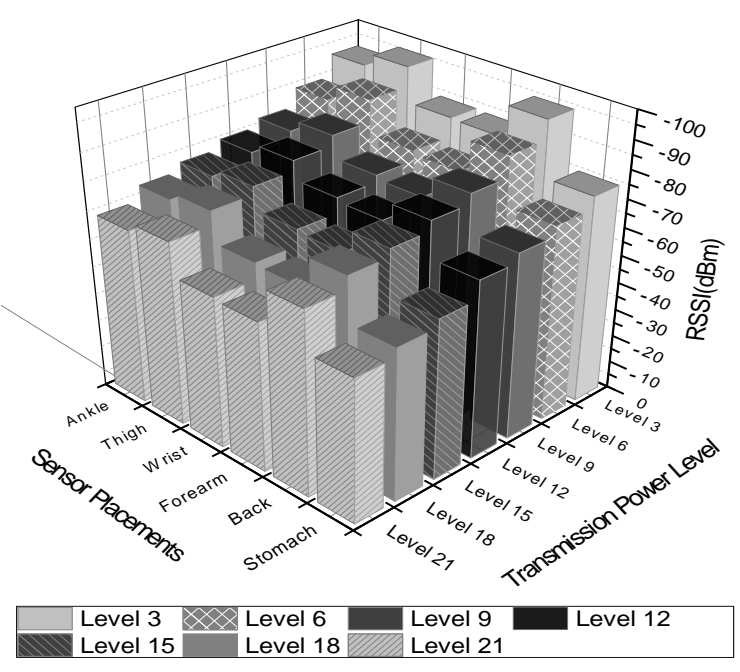

Figure 6. RSSI according to TPL and sensor placement

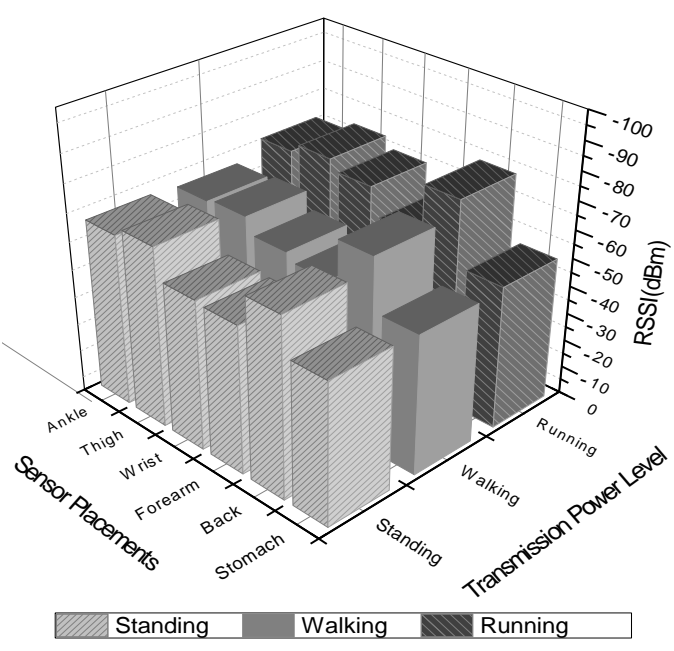

Figure 7. RSSI according to the experimenter's movement and sensor placement

Figure 6 shows the results of the analysis of the channel current based on the placement of the sensors and the TPL. The experimenter was in a "standing" position, and the sensor nodes were placed on his stomach, back, forearm, wrist, thigh, and ankle. Regardless of the site where the sensor nodes were placed, the RSSI value incre ased as the TPL increased. When we compared the RSSI value according to the sensor placement at the same TPL, the order of value size was maintained at the same for other TPL. For example, when TPL was 3, the RSSI value was large descending in the order of the stomach, forearm, wrist, ankle, back, and thigh. Likewise, when the TPL was 21, the size of the RSSI value based on the sensor placement maintained the same order. The results show that the characteristics of each sensor location affect the RSSI value.

Figure 7 shows the different movements of the experimenter and the RSSI value according to the sensor node placement. In this three-dimensional graph, the $\mathrm{x}$ axis indicates the location of the sensor nodes, the y axis indicates the RSSI value, and the $\mathrm{z}$-axis indicates the experimenter's movement. Here, the TPL of the sink node was 22 to increase the packet reception rate and to confirm the effect of the movement and the location of the sensor node by maximizing the transmission power. The RSSI value was higher, on average, in the order of standing > walking > running because channel became more stable as the body movement decreased. For the result based on sensor placement, it was in the same order as that shown in Figure 6: stomach, forearm, wrist, ankle, back, and thigh in a standing position. In the walking and running conditions, the RSSI value was lower when the sensor was located on the subject's back compared to the thigh. The lower value was due to the absence of any great difference in this value when the sensors were placed on the subject's back and thigh in a standing position and to the distance between the sink node and the sensor node on the subject's thigh repeatedly changing during walking and running compared to standing. In addition, when the sensor was located on the subject's back, the channel became unstable and generated multipath fading, resulting in a lower RSSI. 


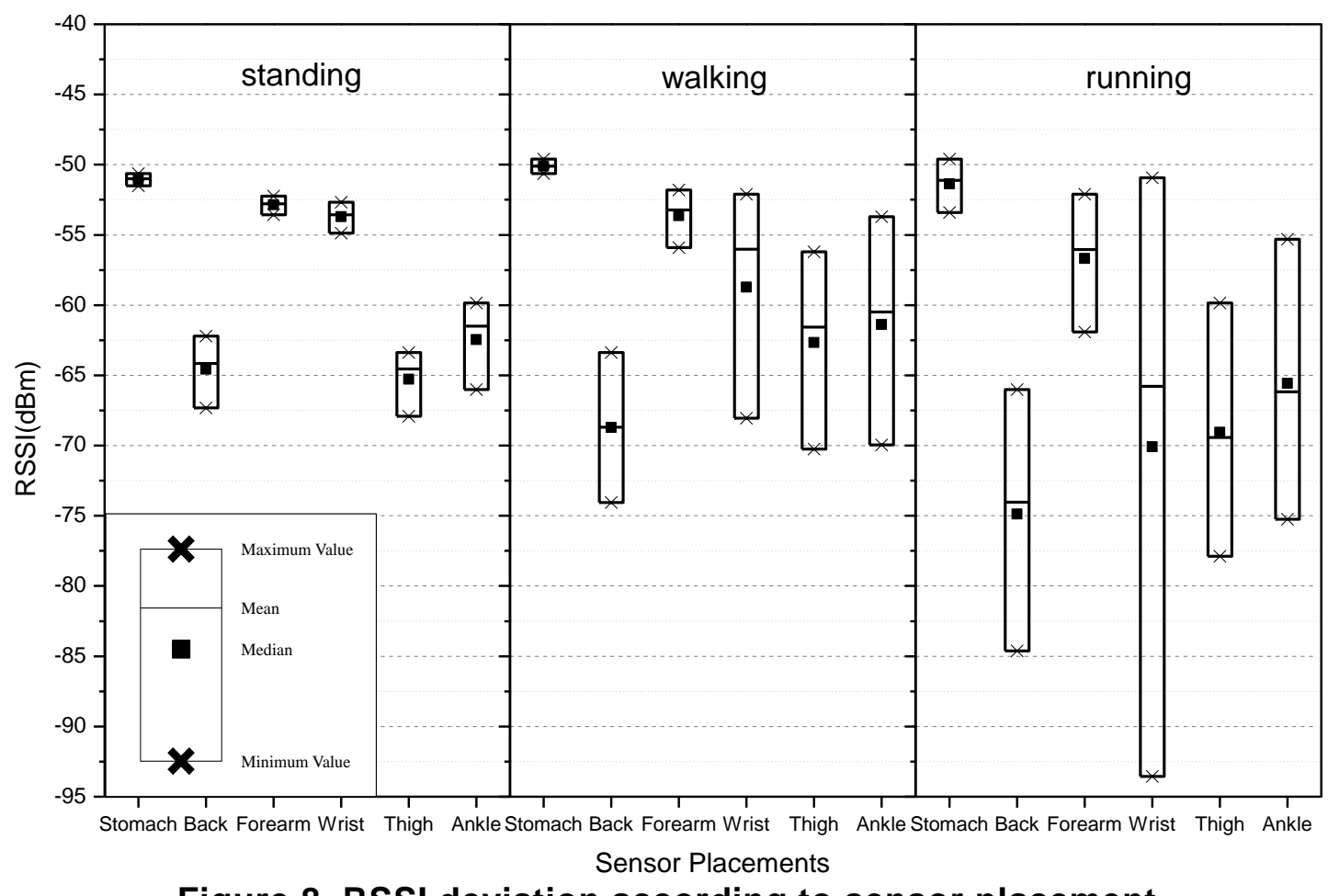

Figure 8. RSSI deviation according to sensor placement

Figure 8 shows the RSSI deviation according to the sensor placement and the experimenter's movement. On each graph, the $\mathrm{x}$ axis indicates the location of the sensor nodes, and the $\mathrm{y}$ axis indicates the RSSI value. The $\mathrm{X}$ mark on the upper side of the rectangle denotes the RSSI max value, the straight line that divides the rectangle denotes the mean value, the square inside the rectangle denotes the median value, and the X mark under the RSSI denotes the RSSI min value. Similar to Figure 7, the sink node TPL was 22. Notably, the RSSI value range increased in all the sensor loc ations as the body movement changed from standing to waking to running. Comparing the standing and running positions, the RSSI value increased at the most 19.4 times and at the least 3.2 times. Based on this result, we can analyze the characteristics of each sensor location.

Table 2. Increase of RSSI deviation

\begin{tabular}{|c|c|c|c|}
\hline \multirow{2}{*}{$\begin{array}{c}\text { Sensor } \\
\text { Placements }\end{array}$} & \multicolumn{3}{|c|}{ Scenarios (times) } \\
\cline { 2 - 4 } & $\begin{array}{l}\text { 1. } \\
\text { Standing : Walking }\end{array}$ & $\begin{array}{l}\text { 2. } \\
\text { Walking : Running }\end{array}$ & $\begin{array}{l}\text { 3. } \\
\text { Standing : Running }\end{array}$ \\
\hline Stomach & 1.2 & 3.7 & 4.3 \\
\hline Back & 2.1 & 1.7 & 3.7 \\
\hline Forearm & 3.1 & 2.4 & 7.4 \\
\hline Wrist & 7.3 & 2.7 & 19.4 \\
\hline Thigh & 3.1 & 1.3 & 4.0 \\
\hline Ankle & 2.6 & 1.2 & 3.2 \\
\hline
\end{tabular}

Table 2 shows the increase in the RSSI deviation of Figure 8 under each scenario. Scenario 1 compared standing and walking, Scenario 2 compared walking and running, 
and Scenario 3 compared standing and running. In the case of the stomach, a static area where the sensor node moves relatively little compared to the other areas, the RSSI deviation was only 1.2 times under Scenario 1. The biggest deviation (3.7 times) was observed under Scenario 2. Based on this result, we can see that body movement has a major influence on the sensor node on the stomach, especially when the experimenter is running because the sensor node is on a line of sight (LOS) with the sink node. In the case of the back, there was little deviation between Scenario 1 and 2, despite this area being static like the stomach. We can conclude that the RSSI value decreased because the back is a non-LOS, far from the sink node, and signal curves occur due to multipath fading and reflection.

The following is an analysis of the dynamic areas: forearm, wrist, thigh, and ankle. As shown in Figure 8 in a standing position, the RSSI value for the forearm was high, and the deviation in the RSSI was small, indicating that the location of the sensor was stable. The increase in the range of RSSI values was greatest for the wrist under Scenario 1. In general, the forearm does not move much when a person walks in a comfortable position, whereas the wrist may move a lot. Unlike Scenario 1, the increase in the range of RSSI values for the forearm and wrist was similar under Scenario 2. This means that the range of movement of the experimenter's forearm and wrist was similar under the walking and running conditions. Likewise, the increase in the range of RSSI values was similar under Scenario 2 because the movement of the thigh and ankle is similar. The deviation in the thigh and ankle under Scenario 2 was 1.3 and 1.2 times, respectively, the smallest of all recorded deviations. This finding is attributable to the sensor locations have similar movement under the walking and running conditions and a similar location to the sensor node accordingly.

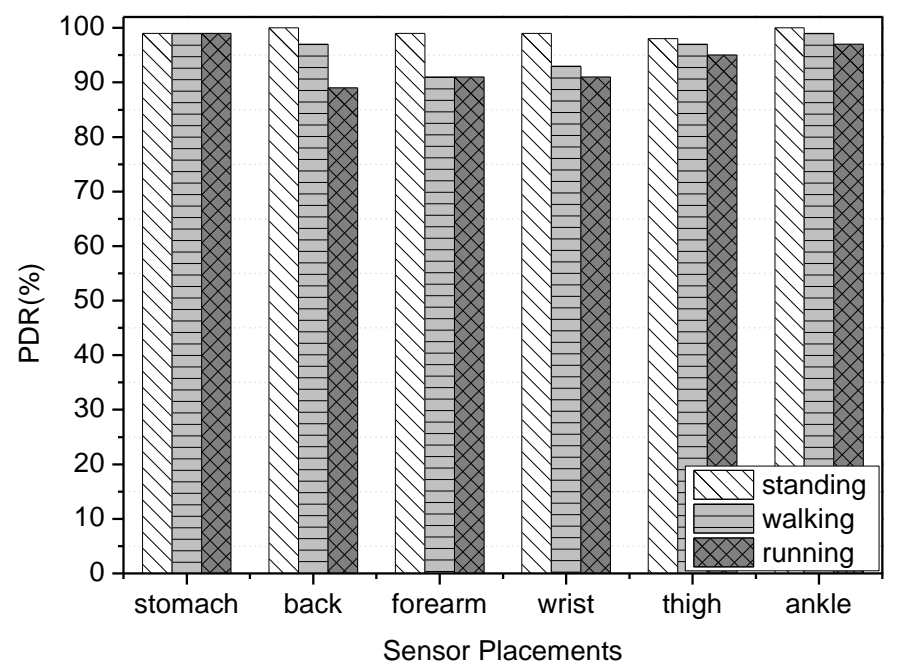
Figure 9. PDR according to sensor placement and the experimenter's
movement

Figure 9 is a bar graph that shows the PDR according to the placement of the sensor nodes. The $\mathrm{x}$ axis indicates the locations of the sensors, and the $\mathrm{y}$ axis shows the PDR. For each sensor, the bar on the left denotes standing, and the middle and right bars denote walking and running, respectively. The TPL was 22 to allow the sink node to transfer at the maximum level. A TPL of 22 is effective for analyzing the effect of the 
locations of the sensors on the experimenter's movement and on the PDR. When the sensor node was on the stomach, the PDR was 99\%, regardless of the experimenter's movement because the sensor node and the sink node are on an LOS. On the other hand, the other sensors on non-LOS showed relatively weaker transmission. When the channel environment was stable, as in the standing position, the PDR was, on average, 99\%. In the walking and running conditions, the PDR was $95 \%$ and $93 \%$, respectively. Although the back is a static area, the PDR was $89 \%$ (the lowest of all the PDRs) due to severe reflection when the experimenter was walking or running. In the case of the forearm and wrist, walking and running generate wobbles and increase the distance between the nodes. As a result, the PDR dramatically dropped compared to the standing position. In common with the RSSI value range, the PDR for the thigh and ankle sensors showed a similar degree of decrease according to the type of movement.

\section{Conclusion}

Various factors affect the RSSI value in WBSNs. This study analyzed the effect of TPL, sensor node placement, and human body movements on the RSSI value and the PDR through actual experiments that alter these factors.

According to the analysis, as the TPL increases, the RSSI value increased, regardless of the location of the sensor node. As the channel is more stable when there is less movement, the RSSI values were in descending order: standing > walking > running. As the experimenter moved from a standing position to walking and running, the range of the RSSI value became wider in all the sensor node locations. Finally, regardless of the experimenter's movements, the PDR was high when the sensor node was on the stomach. However, the transmission rate was relatively low at the other sensor locations.

This study analyzed the characteristics of each sensor location. This analysis of how the movements and the locations of sensor nodes affect the RSSI value and the PDR in a WBSN can be used to support the design of an efficient system for energy consumption. In the future, we plan on developing an algorithm that can help to balance accuracy, latency, and energy overheads in WBSNs.

\section{Acknowledgements}

This research was supported by Basic Science Research Program through the National Research Foundation of Korea (NRF) funded by the Ministry of Education, Science and Technology (grant number 2012R1A1A1002133).

\section{References}

[1] B. Latre, B. Braem, I. Moerman, C. Blondia and P. Demeester, "A Survey on Wireless Body Area Networks"

[2] K. Gosse, "New Radio Interfaces for Short Range Communications", presented at WWRF9, (2003).

[3] H. Cao and V. Leung, "Enabling Technologies for Wireless Body Area Networks: A Survey and Outlook", IEEE Communications Magazine, vol. 47, (2009) December, pp.84-93.

[4] S. Ullah, H. Higgin, M. Arif Siddiqui and K. S. Kwak, "A Study of Implanted and Wearable Body Sensor Networks", KES-AMSTA, (2008) March, pp. 46-47.

[5] X. Qi, G. Zhou, Y. Li and G. Peng, "RadioSense: Exploiting Wireless Communication Patterns for Body Sensor Network Activity Recognition”, IEEE 33rd Real-Time Systems Symposium, (2012), pp. 95-104.

[6] W.S. Lee, M. Choi and N. Kim, "Experimental link channel characteristics in wireless body sensor systems", ICOIN, (2012) February, pp. 374-378.

[7] W. S. Lee, M. Choi and N. Kim, "Different Characteristics of Radio Modules in Wireless Body Sensor Network Systems", Network and Parallel Computing, vol. 7513, (2012) September, pp. 308-314.

[8] A. Natarajan, B. de Silva, K. K. Yap and M. Motani, "Link Layer Behavior of Body Area Networks at 2.4 GHz", MobiCom, (2009) September, pp. 241-252. 
[9] CC2420 Data Sheet, http://inst.eecs.berkeley.edu/ cs150/Documents/CC2420.pdf.

[10] Crossbow-Technology, http://bullseye.xbow.com:81/index.as.

[11] CC1000 Data Sheet, http://www.ti.com/lit/ug/swru058/swru058.pdf.

\section{Authors}

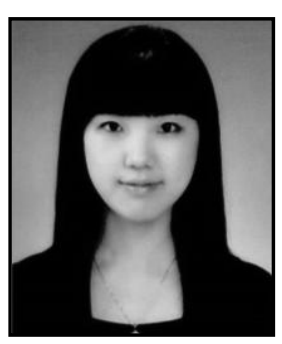

\section{Jina Hong}

She received the B.S. degree in Computer Science from the Kyonggi University, Korea, in 2013. She is currently M.S. candidate in Computer Science from Kyonggi University. Her research interests include wireless systems, sensor networks.

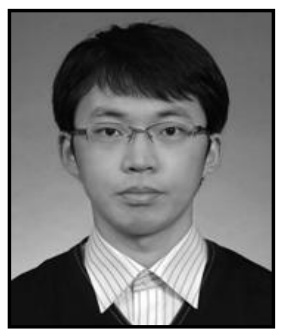

\section{Woosik Lee}

He received the B.S. degree in Computer Science from the Kyonggi University, Korea, in 2009, and the M.S. degree in the Computer Science from Kyonggi University in 2011. He is currently Ph.D. candidate in Computer Science from Kyonggi University. His research interests include wireless systems, sensor networks, and energy management protocols.

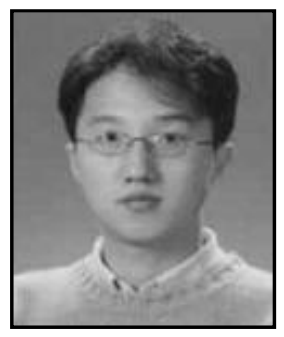

\section{Namgi Kim}

He received the B.S. degree in Computer Science from Sogang University, Korea, in 1997, and the M.S. degree and the Ph.D. degree in Computer Science from KAIST in 2000 and 2005, respectively. From 2005 to 2007, he was a research member of the Samsung Electronics. Since 2007, he has been a faculty of the Kyonggi University. His research interests include sensor system, wireless system, and mobile communication. 
International Journal of Bio-Science and Bio-Technology Vol.6, No.2 (2014) 